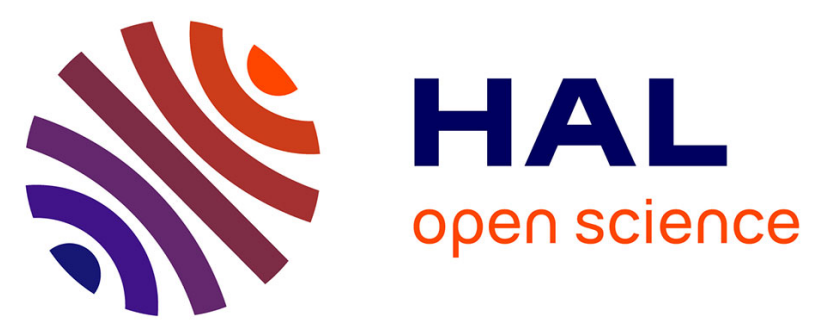

\title{
Personalized mapping of the deep brain with a white matter attenuated inversion recovery (WAIR) sequence at 1.5-tesla: Experience based on a series of 156 patients
}

Abderrahim Zerroug, Jean Gabrillargues, Guillaume Coll, François Vassal, Betty Jean, Emmanuel Chabert, Béatrice Claise, Toufic Khalil, Laurent Sakka, Fabien Feschet, et al.

\section{To cite this version:}

Abderrahim Zerroug, Jean Gabrillargues, Guillaume Coll, François Vassal, Betty Jean, et al.. Personalized mapping of the deep brain with a white matter attenuated inversion recovery (WAIR) sequence at 1.5-tesla: Experience based on a series of 156 patients. Neurochirurgie, 2016, 62 (4), pp.183-189. 10.1016/j.neuchi.2016.01.009 . hal-01578968

\section{HAL Id: hal-01578968 https://hal.science/hal-01578968}

Submitted on 30 Aug 2017

HAL is a multi-disciplinary open access archive for the deposit and dissemination of scientific research documents, whether they are published or not. The documents may come from teaching and research institutions in France or abroad, or from public or private research centers.
L'archive ouverte pluridisciplinaire HAL, est destinée au dépôt et à la diffusion de documents scientifiques de niveau recherche, publiés ou non, émanant des établissements d'enseignement et de recherche français ou étrangers, des laboratoires publics ou privés. 


\title{
Personalized mapping of the deep brain with a white matter attenuated inversion recovery (WAIR) sequence at 1.5-tesla: Experience based on a series of 156 patients
}

\author{
Zerroug A. ${ }^{\mathrm{a}, \mathrm{c}}$, Gabrillargues J., ${ }^{\mathrm{a}, \mathrm{c}}$, Coll G..$^{\mathrm{a}, \mathrm{b}}$, Vassal F. ${ }^{\mathrm{a}}$, Jean B. ${ }^{\mathrm{c}}$, Chabert E. ${ }^{\mathrm{a}, \mathrm{c}}$, \\ Claise B. ${ }^{\mathrm{a}, \mathrm{c}}$, Khalil T. ${ }^{\mathrm{a}, \mathrm{b}}$, Sakka L. ${ }^{\mathrm{a}, \mathrm{b}}$, Feschet F. ${ }^{\mathrm{a}}$, Durif F. ${ }^{\mathrm{d}}$, Boyer L. ${ }^{\mathrm{e}}$, Coste J. ${ }^{\mathrm{a}, \mathrm{b}}$, \\ Lemaire JJ.,
}

a Image-guided clinical neuroscience and connectomics, Clermont université, université d'Auvergne, EA7282, 63000 Clermont-Ferrand, France

b Service of neurosurgery, CHU Gabriel-Montpied, 58, rue Montalembert, 63003 Clermont-Ferrand, France

c Service of radiology, neuroradiology unit, CHU de Clermont-Ferrand, 63003 Clermont-Ferrand, France

d Service of neurology, CHU de Clermont-Ferrand, 63003 Clermont-Ferrand, France

e Service of radiology, CHU de Clermont-Ferrand, 63003 Clemront-Ferrand, France

* Corresponding author : jjlemaire@ chu-clermontferrand.fr

\begin{abstract}
Objective Deep brain mapping has been proposed for direct targeting in stereotactic functional surgery, aiming to personalize electrode implantation according to individual MRI anatomy without atlas or statistical template. We report our clinical experience of direct targeting in a series of 156 patients operated on using a dedicated Inversion Recovery Turbo Spin Echo sequence at 1.5-tesla, called White Matter Attenuated Inversion Recovery (WAIR).

Methods After manual contouring of all pertinent structures and 3D planning of trajectories, 312 DBS electrodes were implanted. Detailed anatomy of close neighbouring structures, whether gray nuclei or white matter regions, was identified during each planning procedure. We gathered the experience of these 312 deep brain mappings and elaborated consistent procedures of anatomical MRI mapping for pallidal, subthalamic and ventral thalamic regions. We studied the number of times the central track anatomically optimized was selected for implantation of definitive electrodes.

Results WAIR sequence provided high-quality images of most common functional targets, successfully used for pure direct stereotactic targeting: the central track corresponding to the optimized primary anatomical trajectory was chosen for implantation of definitive electrodes in $90.38 \%$.
\end{abstract}

Conclusion WAIR sequence is anatomically reliable, enabling precise deep brain mapping and direct stereotactic targeting under routine clinical conditions.

Keywords: MRI, Anatomy, Mapping, Deep brain, DBS, Direct targeting 


\section{Introduction}

Imagery plays a key role in stereotactic functional neurosurgery with the development of electrode implantations for deep brain stimulation (DBS). Classical indirect probabilistic targeting lends to wellknown anatomical imprecision due to inter- and intra-individual variability still tackled [1]. Thus, intraoperative electrophysiological monitoring and/or electrical stimulation compensate limited anatomical errors and account for individual functionality [2]. Advances in anatomical MRI, such as 2D and 3D sequences at 1,1.5 and 3 tesla, refined primary anatomical targeting, revealing stereotactic targets: SWI [3,4], T2WI TSE [5-7], IR TSE [8], IRT1 [9] for the subthalamic nucleus (STN); Proton Density WI SE [10], 3D T1WI GE [11], IR FSE [12,13], T2WI TSE [14], IRT1 [9] for medial, or intern, globus pallidum (GPi); T1WI IR TSE [15] and IRT1 [9] for the ventrointermediate nucleus of thalamus (Vim). Meanwhile, technical issues about direct or mixed (direct and atlas-based) targeting remain unsolved $[16,17]$. On the ground, primary anatomical targeting is still frequently completed by secondary physiological targeting along several trajectories, often three or more, eventually with a second set of trajectories. Because the hemorrhagic risk rises along with the number of tracts, most teams tend to decrease the number of exploratory trajectories [18]. In 2004, we settled a dedicated Inversion Recovery Turbo Spin Echo (IR TSE) sequence, willing to directly spot most deep brain structures; atlas-based coordinates became useless. The sequence optimized on 1.5-Tesla machine (unpublished data), derived from Magnotta et al. [8], was named White Matter Inversion Recovery (WAIR), and is still routinely used. Personalized MRI anatomical mapping was devised for advanced analysis of targets within its anatomic environment, using 3D anatomic objects rendering. This imposed manual contouring of anatomical structures is still mandatory for precise reconstruction of anatomical objects. The aim of our study was to report our clinical experience of deep brain mapping using the WAIR sequence; clinical results have in fact been previously published [19-21].

\section{Case series}

\subsection{Patients and electrodes}

One hundred fifty six patients (mean age $=59 \pm 14$ years) were operated on, five patients for a second surgery due to new long-term severe symptoms: 117, motor complications of severe Parkinson's disease; 20, essential tremor; 12, dystonia; 7, research protocols. Three hundred twelve electrodes were implanted during 137 bilateral (3 with 2 targets) and 14 unilateral procedures: 195 STN targeting within the subthalamic region; 65 Vim targeting within the ventrolateral (motor) thalamus; 39 GPi targeting within the lenticular nucleus; 2 ventro-oral anterior nucleus and 6 center median nuclei of thalamus and 5 GPi targeting for research protocols. Clinical assessments, optimization of electrical settings and medications, and follow-up were carried out by institutional senior neurologists.

\subsection{Stereotactic WAIR sequence}

Routine imaging was performed on Magnetom ${ }^{\mathrm{TM}}$ Sonata and Avanto (Siemens, Erlangen; Germany) 1.5tesla machines (122 times under local anesthesia and 34 times under general anesthesia). The stereotactic frame (Leksell@ Coordinate Frame G, Leksell@ repositioning kit; Sweden) fixed on the patient's head 
with its location box, was locked within the volume head coil, using a specific cradle fixed to the MRI table; the whole setting blocked head motion. Medication was adapted for patient comfort and to minimize any motion. The WAIR sequence was an IR TSE sequence with the following parameters: TE $13 \mathrm{ms,} \mathrm{TR}$ $4500 \mathrm{~ms}$, TI $160 \mathrm{~ms}$, bandwidth $115 \mathrm{~Hz} /$ pixel and turbo factor 7. The images were reconstructed using a magnitude reconstruction algorithm. The 2-mm slice thickness limited signal loss that is observed with thinner slices. We used a large field of view of $270 \mathrm{~mm}$ for visualization of stereotactic fiducials, and a matrix of $512 \times 435$ pixels yielding pixel size of $0.62 \mathrm{~mm} \times 0.52 \mathrm{~mm}$. With our parameters, MRI software precluded a zero-mm slice gap; hence we used a 2-mm distance factor and two interleaved series of 15 slices, moved 2-mm away. This was deemed necessary to optimize calculation of stereotactic frame coordinates and for a good analysis of anatomical stereotactic space. The resulting series of 30 slices lasted $19 \mathrm{~min}$, a duration that was reasonable for the patient. Acquisition was routinely performed in the coronal plan facilitating the identification of anatomic structures merely spread in this plan quasi perpendicular to the rostro-caudal neural axis.

\subsection{Implantation of DBS electrodes}

The 30-image coronal set was transferred to dedicated surgical stereotactic software (iPlan Stereotaxy®, BrainLab, Feldkirchen, Germany). Anatomical structures were contoured and trajectories planned; this lasted about $30 \mathrm{~min}$ to $50 \mathrm{~min}$ for two hemispheres, depending on the number of structures. Double obliquity trajectories entered into the second frontal gyrus, avoided vessels (superficial and deep; spontaneous very low intensity signal, black), caudate nucleus and ventricles, and distally crossed the volume embedding the intended-to-place-contact region (Fig. 1). The day after, the stereotactic frame was repositioned (local anesthesia, 122 times out of 156) and stereotactic CT acquired (frame-based coordinate checking), DBS electrodes (3387@) and 3389๑ leads, Medtronic, Minneapolis, USA) were implanted within the right and/or left hemisphere(s). Electrophysiological recordings and/or stimulation tests

optimized the determination of site for definitive electrode-contact positioning, using for each hemisphere two exploration electrodes (FHC Word precision instruments Inc., Sarasota FL, USA; Alpha Omega, Israel) with two guide tubes (Bengun, Immi; France): one on the anatomically optimized planned tract (central) and one on a 2-mm distant and parallel tract. CT was performed after removal of the stereotactic frame, checking electrode location and searching for surgical complications. 


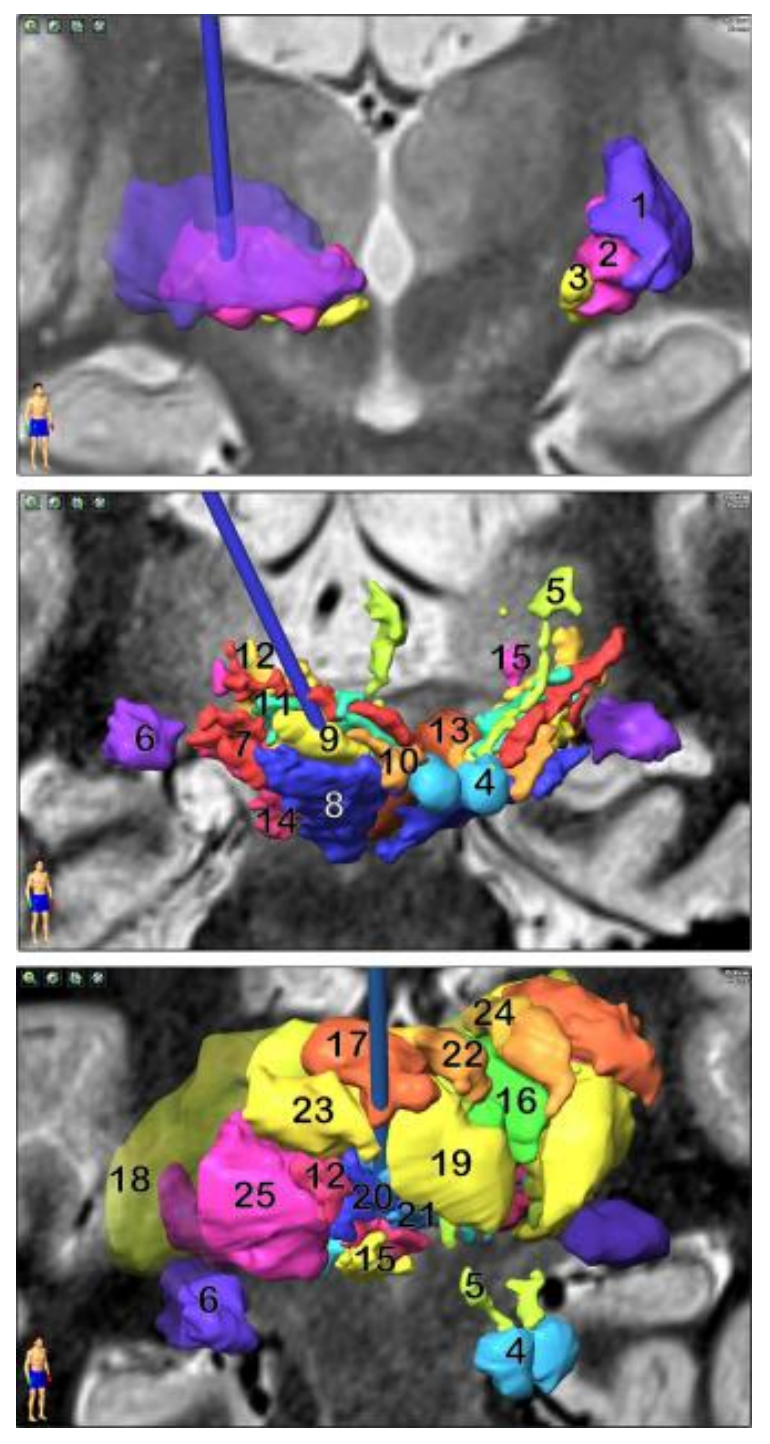

Figure 1. Planning of primary anatomical 3D personalized targeting. $3 D$ objects, built from the contours of anatomical structures, are displayed (right anterolateral views) on WAIR coronal slices (interpolated). The trajectories optimized for primary anatomical $3 D$ personalized targeting go through the stereotactic targets: top, medial pallidum, at the junction between the posterior and intermediate third of the nucleus (1: lateral pallidum; 2: medial pallidum; 3: intern-medial pallidum); intermediate, the subthalamic nucleus, at the superior and lateral part (sensorimotor) of the nucleus, as well as, immediately above, the zona incerta and Forel's fields (4: mammillary body; 5: mammillo-thalamic bundle; 6: lateral geniculate body; 7: zona incerta; 8: substantia nigra; 9: subthalamic nucleus; 10: nucleus of ansa lenticularis; 11: Forel's fields; 12: nucleus ventrocaudal medial of the thalamus; 13: red nucleus; 14: peripeduncular nucleus; 15: lemniscal fascicle); bottom, the ventrointermediate nucleus of thalamus (thalamic nuclei: 16: median; 17: dorsolateral; 18: pulvinar; 19: anterolateral; 20: ventrointermediate; 21: ventro-oral; 22: anteromedial; 23: intermediate; 24: dorsomedial; 25: ventrocaudal lateral). 


\subsection{Deep brain mapping}

Manual contouring was performed on raw images, without signal-intensity interpolation and slice reorientation. Basically, WAIR sequence generated low intensity and high intensity signal, respectively for white matter and gray matter structures. Contouring was carried on according to signal intensity of each structure and contrast between neighboring structures, with test-retest and inferential contouring, and two-times processing, broad contouring at small scale followed by precise contouring at large scale (Fig. 2). Each contoured structure became a labeled object, matching classical knowledge obtained from anatomy books and stereotactic atlases, and fitting with a $4.7 \mathrm{MRI}$ atlas providing similar contrasts to those of WAIR images [22]. Contours (color coded) of objects were overlaid on interpolated images and used for 3D anatomical control and optimization of trajectories; this facilitated the understanding of anatomy whatever the orientation, especially along non-standard reconstructed trajectory plans (Fig. 3).

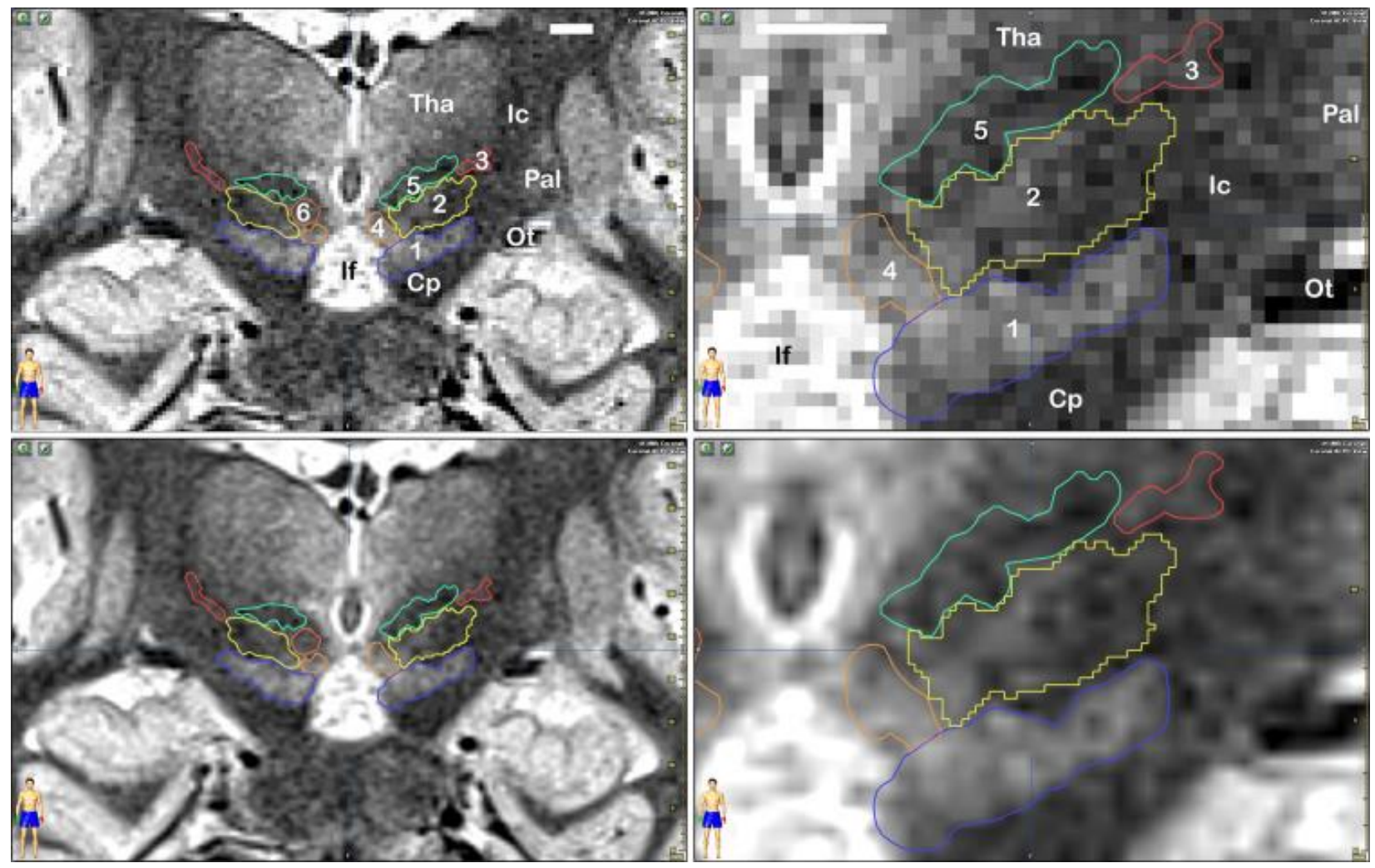

Figure 2. Principle of manual contouring. Anatomical subthalamic structures (white bar $=5 \mathrm{~mm}$; left column, overview; right column, large scale; pixel size $0.62 \mathrm{~mm} \times 0.52 \mathrm{~mm}$ ) contoured on WAIR coronal image going through the subthalamic nucleus (broken line); each contour was drawn on raw image (top row), interpolated image is given for comparison (bottom row): 1: substantia nigra; 2: subthalamic nucleus; 3: zona incerta; 4: nucleus of ansa lenticularis; 5: Forel's fields; 6: red nucleus; 7: substance Q; 8: mammillo-thalamic bundle; 9: lateral geniculate body; 10: peripeduncular nucleus; 11: mammillary body; V3: 3rd ventricle; Pal: pallidum; Ic: internal capsule; Cp: cerebral peduncle; If: inter-peduncular fossa. 


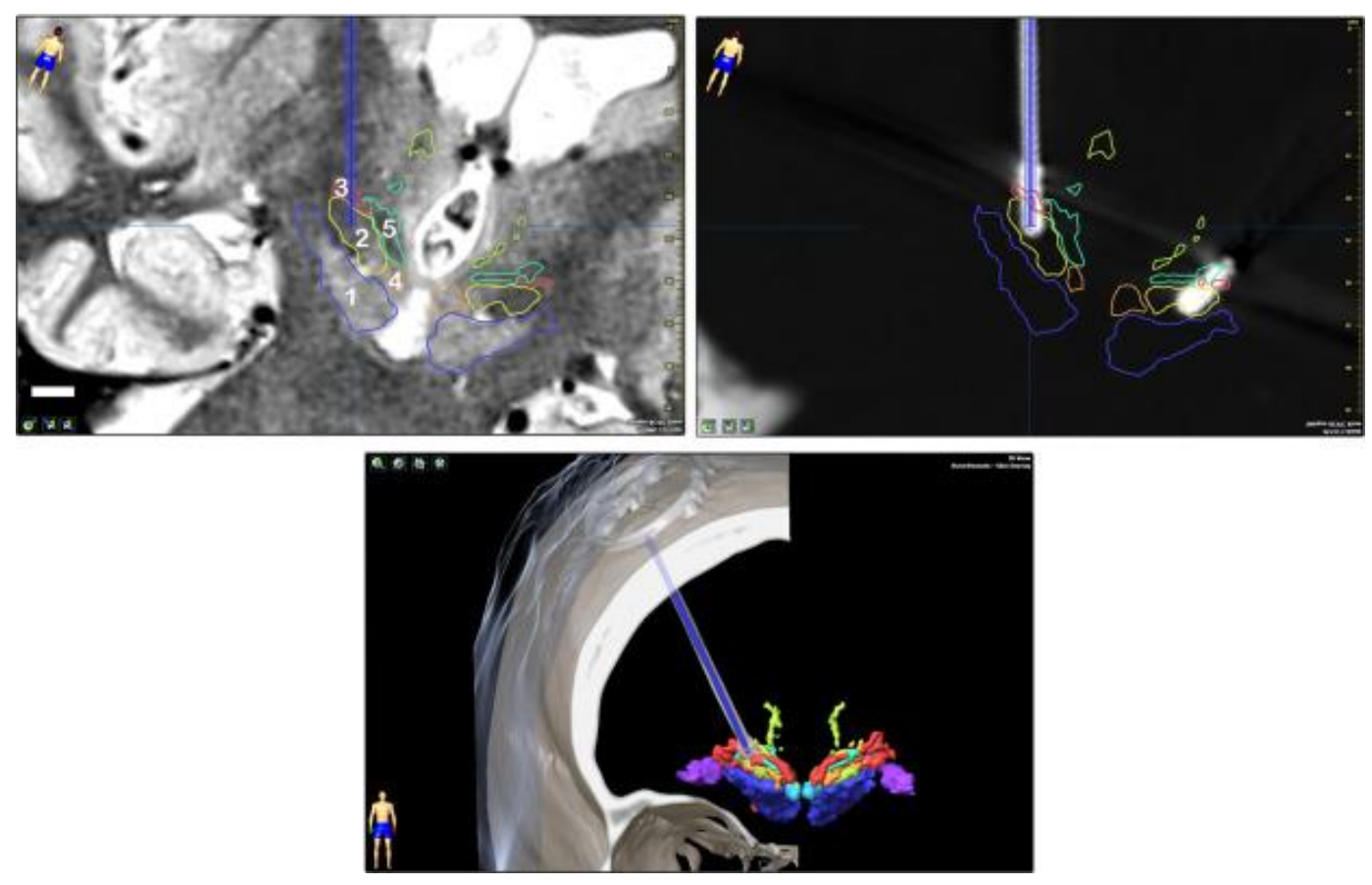

Figure 3. Reconstructed images and objects. Anatomical objects overlaid on interpolated reconstructed WAIR image along trajectory (top; planned trajectory is blue), anatomical objects overlaid on interpolated reconstructed CT-scan (high energy) image along electrode (intermediate), and 3D rendering of objects with planned trajectory and electrode and bone reconstructions (thresholding segmentation); right-hemisphere subthalamic targeting: 1: substantia nigra; 2: subthalamic nucleus; 3 : zona incerta; 4: nucleus of ansa lenticularis; 5: Forel's fields.

\subsection{Analysis}

We used the experience of the 312 deep brain mappings gained during the 156 surgical procedures and elaborated consistent procedures of brain mapping for pallidal, subthalamic and ventral thalamic regions. We also studied the number of times the central track anatomically optimized was selected for the definitive implantation of DBS leads.

\section{Results}

\subsection{MRI mapping of lenticular, subthalamic and ventral thalamic regions}

Standard mapping of lenticular region is exemplified in Fig. 4. Striatal structures, putamen, caudate, accumbens nucleus and sublenticular nuclei, were visible, as well as trabeculae of cell islands resulting of the dissociation of caudate and putamen neurons by cortico-fugal projection fibers. 


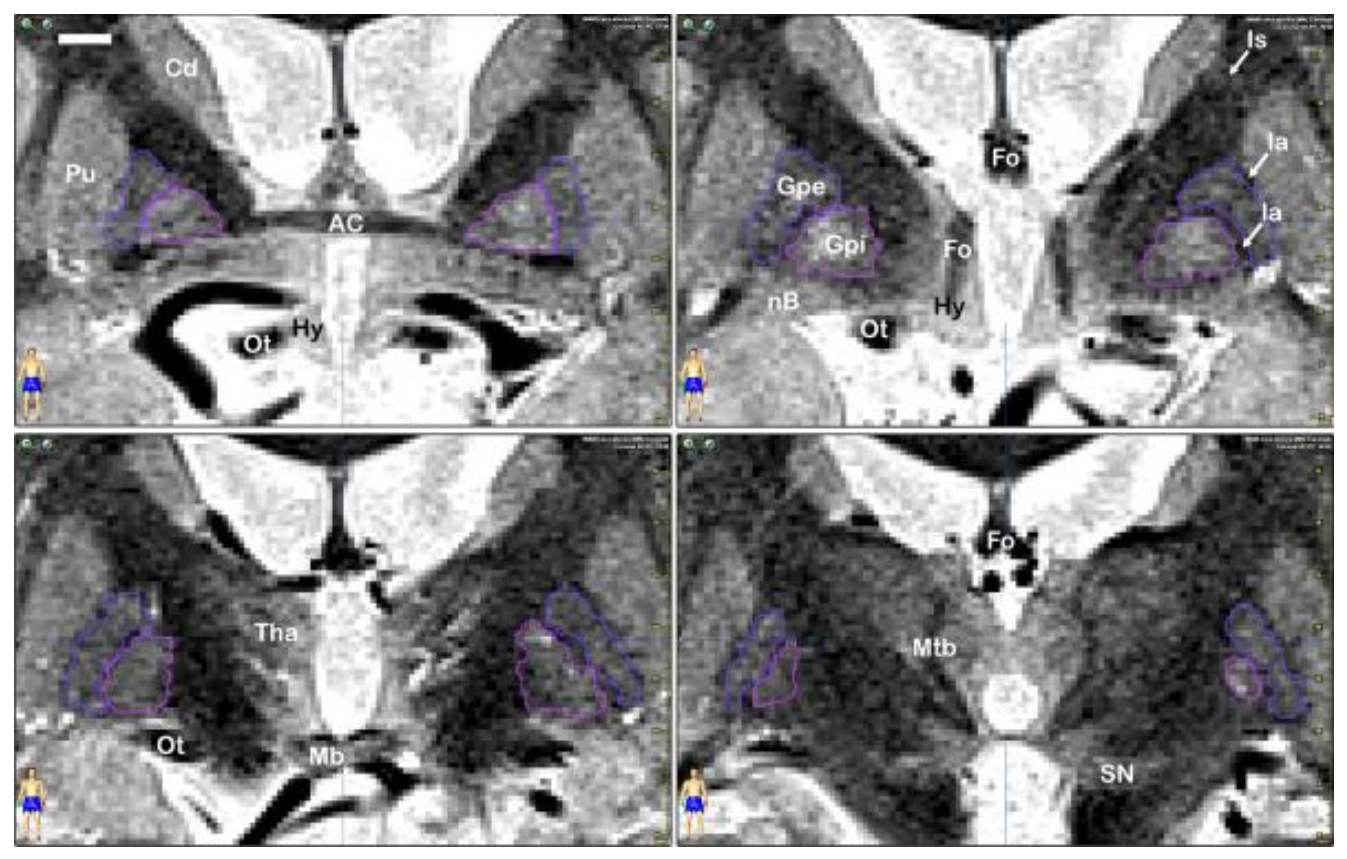

Figure 4. Lenticular mapping. Contours of anatomical structures of the lenticular region overlaid over raw WAIR images (white bar $=5 \mathrm{~mm}$ ) from anterior (top left) to posterior (bottom right), slice distance 4 mm: AC: anterior commissure; $C d$ : caudate; $C$ : cerebral peduncle; Ec: external and extreme capsules; Fo: fornix; GPe: external pallidum; GPi: medial pallidum; Hy: hypothalamus; Is: islands of cells; la: lamina; Mtb: mammillo-thalamic bundle; Ot: optical tract; Pu: putamen; SN: substantia nigra.

The sublenticular extension of the internal capsule lined the ventral pallidum above, and sublenticular nuclei below. Extern or intern (GPi; with the intern-lateral and intern-medial subparts) pallidum, either, had lower signal intensity than striatum, notably postero-medially, because of the high density of radiate fibers [23]; the low signal intensity was maximum within the intern-medial pallidum containing the origin of "faisceau pallidal de la pointe" [24]. The different lenticular components were clearly separated by lamina, lateral and medial, containing white matter bundles of myelinated axonal fibers, notably those of the ansa lenticularis [25].

Almost all subthalamic structures were visible (Fig. 5): substantia nigra, STN, zona incerta, nucleus of ansa lenticularis (putamen rubral), mammillary bodies, fields of Forel, mammillo-thalamic (Vicq d'Azyr) fascicle or bundle, red nucleus, substance $\mathrm{Q}$, and neighboring structures, such as peri peduncular nucleus and lateral geniculate body. 


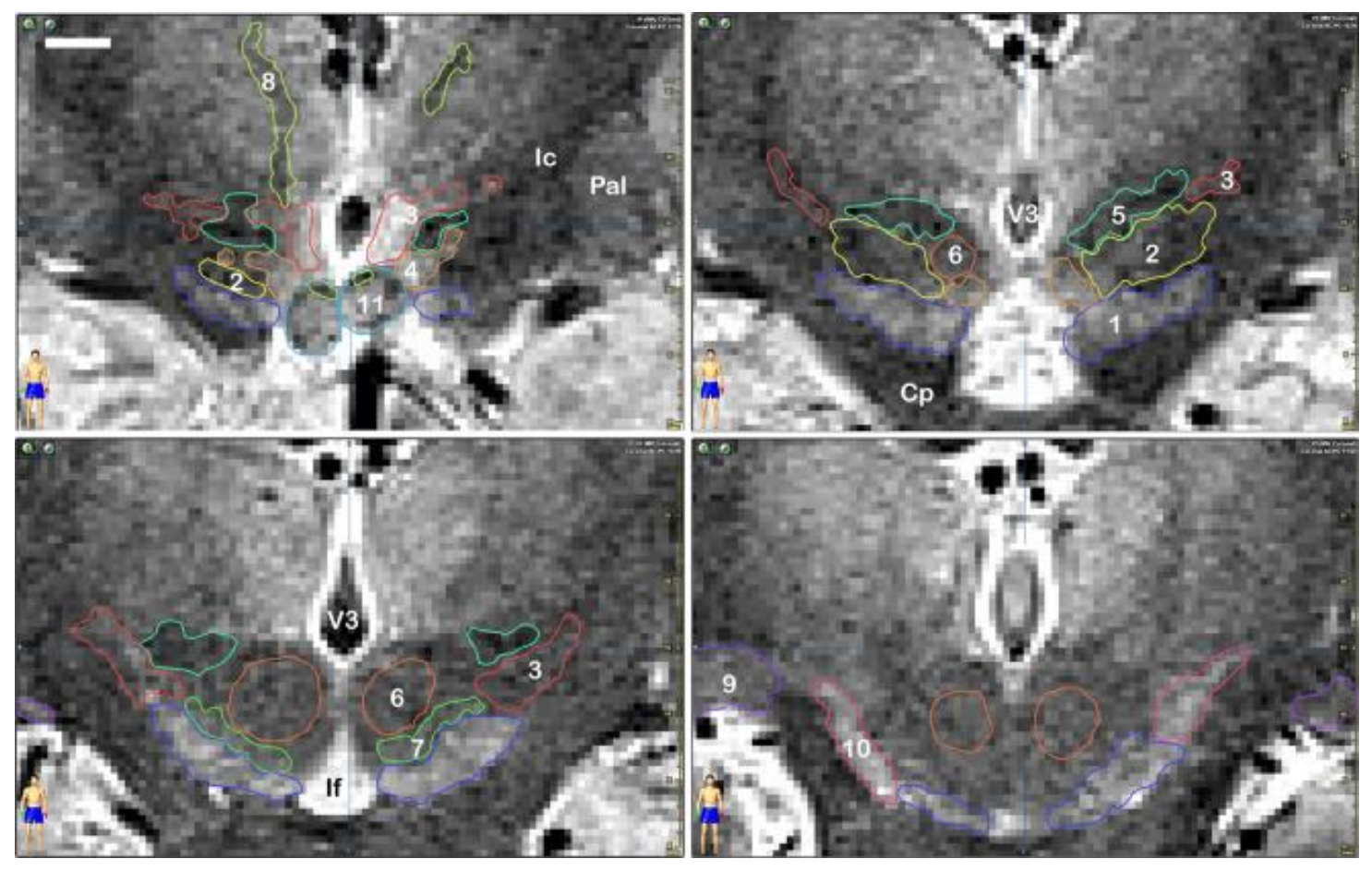

Figure 5. Subthalamic mapping. Contours of anatomical subthalamic structures overlaid over raw WAIR images (white bar $=5 \mathrm{~mm}$ ) from anterior (top left) to posterior (bottom right), slice distance $4 \mathrm{~mm}$ : 1 : substantia nigra; 2: subthalamic nucleus; 3: zona incerta; 4: nucleus of ansa lenticularis; 5: Forel's fields; 6: red nucleus; 7: substance Q; 8: mammillo-thalamic bundle; 9: lateral geniculate body; 10: peripeduncular nucleus; 11: mammillary body; V3: 3rd ventricle; Pal: pallidum; Ic: internal capsule; Cp: cerebral peduncle; If: inter-peduncular fossa.

The nucleus of ansa lenticularis spanning the subthalamus and the sublenticular region [25] had very high signal intensity, more anteriorly than posteriorly. Arnold's net and substance Q had high signal intensity and were in continuity with zona incerta, respectively dorsally and ventrally. The intermediate part of zona incerta was not clearly identified, except sparse cell islands separated by very low signal intensity spots of bundles of myelinated axons belonging to the superior thalamic radiations crossing the Arnold's net (reticular nucleus of thalamus) and zona incerta [26]. The STN had medium, inhomogeneous, signal intensity, higher anteriorly and superiorly. The red nucleus had low signal intensity partially due to the homogenous and dense distribution of white matter fibers crossing the nucleus, notably those coming through the brachium conjunctivum [25]. It was surrounded by very low signal intensity parcels of myelinated axonal fibers of small white matter fascicles, such as the mammillo-tegmental tract and the longitudinal dorsal tract [25]. The mammillary bodies had a high intensity signal and contained superiorly, medially the mammillo-thalamic fasciculus, and laterally the fornix. $\mathrm{H}$ field of Forel, containing prelemniscal radiations with the central tegmental tract [24], formed a large low intensity area beneath the thalamus. 
The entire signal intensity of thalamus was very inhomogeneous as it is made up of numerous nuclei and white mater laminas. Eight groups of nuclei, anterior or oral, dorsal, intermediate, ventral, medial, laminar, posterior or caudal and superficial were identified [19], according to MRI terminology developed to simplify labels; using classical terminologies render the orientation within the thalamus sometimes confusing [22]. The low signal intensity of Vim was almost always seen within only one coronal slice as it is very thin, assuming that its hypo intense signal was due to the numerous bundles of myelinated axons crossing it [24], notably those of the cerebello-rubro-thalamic tract (Fig. 6).

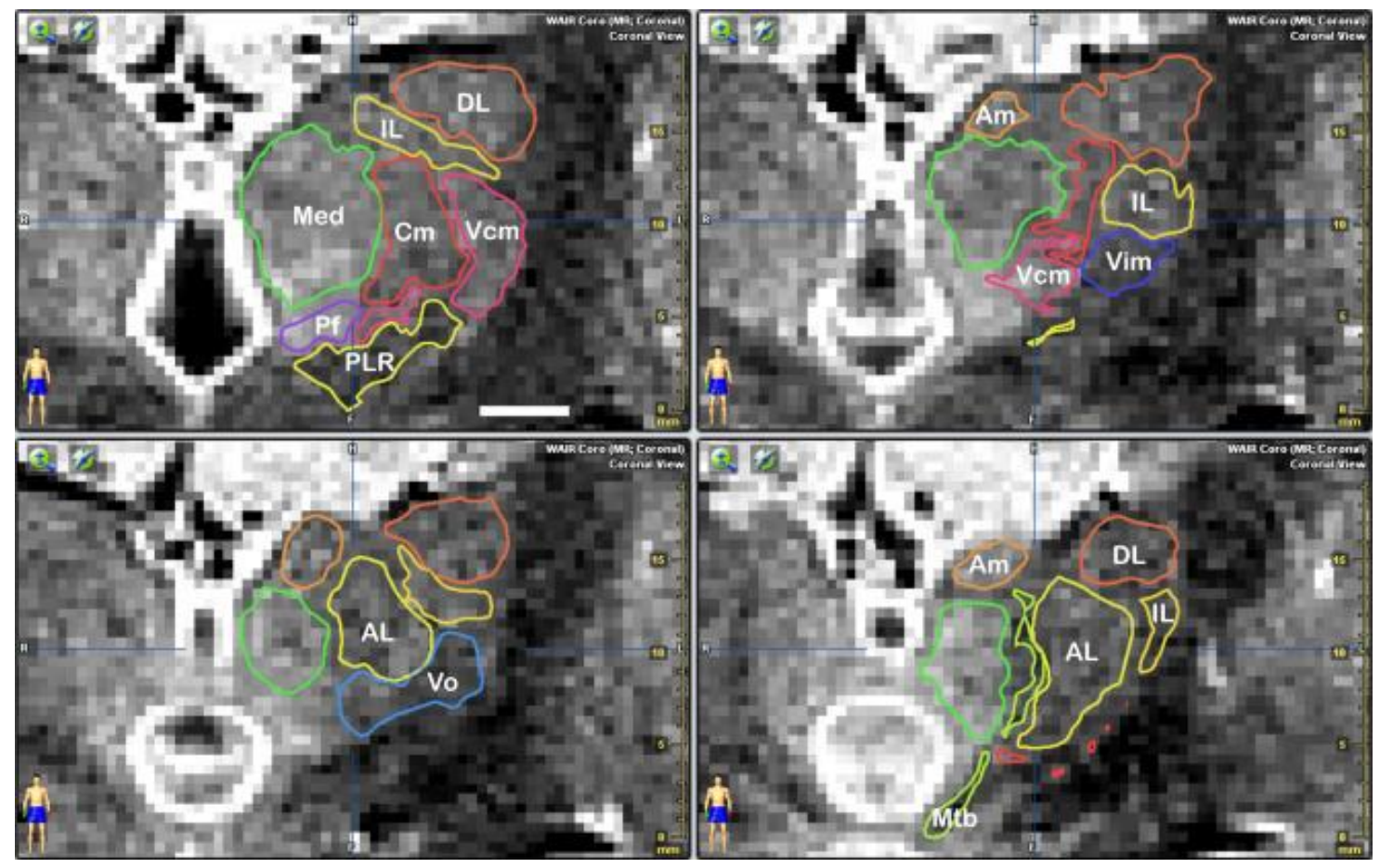

Figure 6. Thalamic mapping. Contours of thalamic nuclei, prelemniscal radiations (PLR), and mammillothalamic bundle $(M t b)$ overlaid over raw WAIR images (white bar $=5 \mathrm{~mm}$ ) from posterior (top left) to anterior (bottom right), slice distance $2 \mathrm{~mm}$ : AL: anterolateral nucleus; Am: anteromedial nucleus; IL: intermediolateral nucleus; Cm: centromedian; Pf: parafascicular; DL: dorsolateral; Vim: ventrointermediate; Vo: ventro-oral; Med: median.

\subsection{Selection of definitive DBS lead implantation tract according to planned trajectories}

The central tract corresponding to the optimized anatomical trajectory was chosen for definitive implantation of DBS leads, 282 times out of 312, i.e. for $90.38 \%$ of electrodes: 37 times out of 39 (94.87\%) for GPi targeting, 180 times out of 195 (92.31\%) for STN targeting, and 50 times out of 63 (79.37\%) for Vim targeting. 


\section{Discussion}

This 10-year clinical experience of DBS surgery relying on the WAIR sequence shows that most deep brain structures are identifiable, under routine conditions, at 1.5 tesla. This anatomical information is reliable for pure direct stereotactic targeting, minimizing the number of exploratory trajectories, shortening surgical time and participating to the reduction of hemorrhagic risk. The overall primary anatomical targeting for the three most common targets led to a correct location of electrodes on average in $90.38 \%$ of cases. The best correspondence between anatomy and DBS was in the GPi and STN, although the STN itself is not the only effective functional target, e.g. for Parkinson's disease [27]. The VIM alone is clearly not the sole structure involved in tremor control, as suggested as from 1978 [28] and confirmed by our results since "only" almost $80 \%$ of primary anatomic targeting was used for definitive lead implantation. It is noticeable that patients requiring a second DBS surgery for new symptoms, do not have to undergo a second MRI acquisition; the second mapping is feasible on the same WAIR image set co-registered with an updated high-resolution stereotactic CT (5 patients in our series). This is also interesting since MRI after electrode implantation is extremely constrained (http://www.medtronic.com/physician/activa/), even if the risks could be overestimated [29]. Furthermore, the extended visualization of the detailed anatomy of deep brain, make this sequence interesting for other highly functional structures [30]. However, precise MRI mapping still remains meticulous and time consuming, particularly for contouring and 3D planning. This requires macro- and micro-anatomical knowledge, which is not still widely spread among neuroradiologists, neurosurgeons, neurologists and clinical neuroscientists. Very high-field MRI atlases are helpful, notably when they provide contrasts close to those obtained under clinical conditions, supplying more precise data [31,32] and also full 3D organization of labeled objects [22].

The WAIR sequence is above all an anatomical imaging able to contrast deep brain structures during an acceptable time of acquisition (with precautions for limiting head motion) under routine clinical conditions. The short TI (160 ms), explored by other teams [13,14,33], and the magnitude reconstruction algorithm are the main parameters affecting signal intensity. Thus signal intensity of nuclei, such as pallidum, red nucleus, Vim, centromedian nucleus of thalamus and STN, was lowered according to the additional component of white matter.

\section{Conclusion}

Under clinical conditions, with the 1.5-tesla MRI machine, and an optimized IR TSE sequence, a large quantity of very accurate anatomic details of the deep brain are harvested, useable for pure direct targeting in functional stereotactic surgery, and beyond for topographical analysis of effective contacts location. On the viewing angle of advanced medical imaging centers, very high-field MRI scanner appears promising for pure direct targeting and anatomical mapping [34,35]. Meanwhile, improvement of sequences at 1.5 and 3 tesla should be useful. Refinement of the MRI technique is also expected to secure and to improve post implantation scanning [36], enabling precise structural and functional analysis of DBS. Improvement of deep brain mapping passes through the spreading of 3D anatomical knowledge tackled by most teams working on direct MRI guiding. Diffusion Tensor Imaging fiber tracking, augmenting the anatomical content, seems promising [37,38]. In parallel further clinical applications should benefit of advance of 
medical software, simplifying notably contrast-based delineation of structures, making easier and faster mapping, whatever the clinical application.

\section{Disclosure of interest}

The authors declare that they have no competing interest.

\section{Acknowledgments}

Abderrahim Zerroug had a financial support from the CHU of Clermont-Ferrand (6-month contract of research; 2014; master degree; M2 TechMed; Auvergne University).

\section{References}

[1] J. Anthofer, K. Steib, C. Fellner, M. Lange, A. Brawanski, J. Schlaier The variability of atlas-based targets in relation to surrounding major fibre tracts in thalamic deep brain stimulation Acta Neurochir (Wien), 156 (8) (2014), pp. 1497-1504

[2] Z. Israel, K.J. Burchiel Microelectrode recording in movement disorder surgery Thieme, New York (2004) [220 p.]

[3] A.T. Vertinsky, V.A. Coenen, D.J. Lang, S. Kolind, C.R. Honey, D. Li, et al. Localization of the subthalamic nucleus: optimization with susceptibility-weighted phase MR imaging AJNR Am $J$ Neuroradiol, 30 (9) (2009), pp. 1717-1724

[4] M. Lefranc, S. Derrey, P. Merle, M. Tir, J.-M. Constans, D. Montpellier, et al. High-resolution 3dimensional T2*-weighted angiography (HR 3-D SWAN): an optimized 3-T magnetic resonance imaging sequence for targeting the subthalamic nucleus Neurosurgery, 74 (6) (2014), pp. 615-626

[5] K.V. Slavin, K.R. Thulborn, C. Wess, H. Nersesyan Direct visualization of the human subthalamic nucleus with 3 T MR imaging AJNR Am J Neuroradiol, 27 (1) (2006), pp. 80-84 
[6] B.P. Bejjani, D. Dormont, B. Pidoux, J. Yelnik, P. Damier, I. Arnulf, et al. Bilateral subthalamic stimulation for Parkinson's disease by using three-dimensional stereotactic magnetic resonance imaging and electrophysiological guidance J Neurosurg, 92 (4) (2000), pp. 615-625

[7] P. Plaha, N.K. Patel, S.S. Gill Stimulation of the subthalamic region for essential tremor J Neurosurg, 101 (1) (2004), pp. 48-54

[8] V.A. Magnotta, S. Gold, N.C. Andreasen, J.C. Ehrhardt, W.T. Yuh Visualization of subthalamic nuclei with cortex attenuated inversion recovery MR imaging Neuroimage, 11 (4) (2000), pp. 341-346

[9] A. Sudhyadhom, I.U. Haq, K.D. Foote, M.S. Okun, F.J. Bova A high resolution and high contrast MRI for differentiation of subcortical structures for DBS targeting: the Fast Gray Matter Acquisition T1 Inversion Recovery (FGATIR) Neuroimage, 47 (Suppl. 2) (2009), pp. T44-T52

[10] J.J. Lemaire, F. Durif, J.Y. Boire, B. Debilly, B. Irthum, J. Chazal Direct stereotactic MRI location in the globus pallidus for chronic stimulation in Parkinson's disease Acta Neurochir (Wien), 141 (7) (1999), pp. 759-765

[11] N. Vayssiere, S. Hemm, M. Zanca, M.C. Picot, A. Bonafe, L. Cif, et al. Magnetic resonance imaging stereotactic target localization for deep brain stimulation in dystonic children J Neurosurg, 93 (5) (2000), pp. 784-790

[12] M.O. Pinsker, J. Volkmann, D. Falk, J. Herzog, K. Alfke, F. Steigerwald, et al. Electrode implantation for deep brain stimulation in dystonia: a fast spin-echo inversion-recovery sequence technique for direct stereotactic targeting of the GPI Zentralbl Neurochir, 69 (2) (2008), pp. 71-75

[13] C.A. Reich, P.A. Hudgins, S.K. Sheppard, P.A. Starr, R.A. Bakay A high-resolution fast spin-echo inversion-recovery sequence for preoperative localization of the internal globus pallidus AJNR Am $J$ Neuroradiol, 21 (5) (2000), pp. 928-931

[14] P.A. Starr, J.L. Vitek, M. DeLong, R.A. Bakay Magnetic resonance imaging-based stereotactic localization of the globus pallidus and subthalamic nucleus Neurosurgery, 44 (2) (1999), pp. 303-313 
[15] A.A. Patil, T. Falloon, F. Hahn, J. Cheng, S. Wang Direct identification of ventrointermediate nucleus of the thalamus on magnetic resonance and computed tomography images Surg Neurol, 51 (6) (1999), pp. 674-678

[16] R.A. Vega, K.L. Holloway, P.S. Larson Image-guided deep brain stimulation Neurosurg Clin N Am, 25 (1) (2014), pp. 159-172

[17] K.A. Sillay, D. Rusy, L. Buyan-Dent, N.L. Ninman, K.K. Vigen Wide-bore 1.5 T MRI-guided deep brain stimulation surgery: initial experience and technique comparison Clin Neurol Neurosurg, 127 (2014), pp. 79-85

[18] L. Zrinzo, T. Foltynie, P. Limousin, M.I. Hariz Reducing hemorrhagic complications in functional neurosurgery: a large case series and systematic literature review J Neurosurg, 116 (1) (2012), pp. 84-94

[19] F. Vassal, J. Coste, P. Derost, V. Mendes, J. Gabrillargues, C. Nuti, et al. Direct stereotactic targeting of the ventrointermediate nucleus of the thalamus based on anatomic 1.5-T MRI mapping with a white matter attenuated inversion recovery (WAIR) sequence Brain Stimul, 5 (4) (2012), pp. 625-633

[20] F. Caire, P. Derost, J. Coste, J.-M. Bonny, F. Durif, E. Frenoux, et al. [Subthalamic deep brain stimulation for severe idiopathic Parkinson's disease. Location study of the effective contacts] Neurochirurgie, 52 (1) (2006), pp. 15-25

[21] A. Marques, O. Chassin, D. Morand, B. Pereira, B. Debilly, P. Derost, et al. Central pain modulation after subthalamic nucleus stimulation: a crossover randomized trial Neurology, 81 (7) (2013), pp. 633-640

[22] J. Lemaire, L. Sakka, L. Ouchchane, F. Caire, J. Gabrillargues, J. Bonny Anatomy of the human thalamus based on spontaneous contrast and microscopic voxels in high-field magnetic resonance imaging Neurosurgery, 66 (3 Suppl. Operative) (2010), pp. 161-172

[23] J. Dejerine Anatomie des centres nerveux, Vol. 1 and 2 Rueff et Cie, Paris (1901) 
[24] J. Talairach, M. David, P. Tournoux, H. Corredor, T. Kvasina Atlas d'anatomie stéréotaxique. Repérage radiologique indirect des noyaux gris centraux des régions mésencéphalo-sous-optiques et hypothalamiques de l'homme Masson et Cie, Paris (1957)

[25] H. Riley An atlas of the basal ganglia, brain stem and spinal cord Williams \& Wilkins, Baltimore (1953) [708 p.]

[26] J.-J. Lemaire, G. Cosnard, L. Sakka, C. Nuti, W. Gradkowski, S. Mori, et al. White matter anatomy of the human deep brain revisited with high resolution DTI fibre tracking Neurochirurgie, 57 (2) (2011), pp. 52-67

[27] F. Velasco, F. Jiménez, M.L. Pérez, J.D. Carrillo-Ruiz, A.L. Velasco, J. Ceballos, et al. Electrical stimulation of the prelemniscal radiation in the treatment of Parkinson's disease: an old target revised with new techniques Neurosurgery, 49 (2) (2001), pp. 293-306

[28] P.J. Kelly, P. Derome, G. Guiot Thalamic spatial variability and the surgical results of lesions placed with neurophysiologic control Surg Neurol, 9 (5) (1978), pp. 307-315

[29] L. Zrinzo, F. Yoshida, M.I. Hariz, J. Thornton, T. Foltynie, T.A. Yousry, et al. Clinical safety of brain magnetic resonance imaging with implanted deep brain stimulation hardware: large case series and review of the literature World Neurosurg, 76 (1-2) (2011), pp. 164-172

[30] J.-J. Lemaire, H. Nezzar, L. Sakka, Y. Boirie, D. Fontaine, A. Coste, et al. Maps of the adult human hypothalamus Surg Neurol Int, 4 (Suppl. 3) (2013), pp. S156-S163

[31] Z.-H. Cho 7. 0 Tesla MRI brain atlas: in vivo atlas with cryomacrotome correlation Springer Science \& Business Media (2010) [567 p.]

[32] T.P. Naidich, H.M. Duvernoy, B.N. Delman, A.G. Sorensen, S.S. Kollias, E.M. Haache Duvernoy's atlas of the human brain stem and cerebellum Springer Wien New York, Austria (2009) [876 p.] 
[33] H. Oikawa, M. Sasaki, Y. Tamakawa, S. Ehara, K. Tohyama The substantia nigra in Parkinson disease: proton density-weighted spin-echo and fast short inversion time inversion-recovery MR findings AJNR Am J Neuroradiol, 23 (10) (2002), pp. 1747-1756

[34] A. Abosch, E. Yacoub, K. Ugurbil, N. Harel An assessment of current brain targets for deep brain stimulation surgery with susceptibility-weighted imaging at 7-tesla Neurosurgery, 67 (6) (2010), pp. 17451756

[35] Z.-H. Cho, H.-K. Min, S.-H. Oh, J.-Y. Han, C.-W. Park, J.-G. Chi, et al. Direct visualization of deep brain stimulation targets in Parkinson disease with the use of 7 -tesla magnetic resonance imaging $J$ Neurosurg, 113 (3) (2010), pp. 639-647

[36] S.N. Sarkar, E. Papavassiliou, D.B. Hackney, D.C. Alsop, L.C. Shih, A.J. Madhuranthakam, et al. Three-dimensional brain MRI for DBS patients within ultra-low radiofrequency power limits Mov Disord, 29 (4) (2014), pp. 546-549

[37] J.-J. Lemaire, J. Coste, L. Ouchchane, F. Caire, C. Nuti, P. Derost, et al. Brain mapping in stereotactic surgery: a brief overview from the probabilistic targeting to the patient-based anatomic mapping Neuroimage, 37 (Suppl. 1) (2007), pp. S109-S115

[38] I. Kovanlikaya, L. Heier, M. Kaplitt Treatment of chronic pain: diffusion tensor imaging identification of the ventroposterolateral nucleus confirmed with successful deep brain stimulation Stereotact Funct Neurosurg, 92 (6) (2014), pp. 365-371 